La communication de recrutement a l'université

Laval. Une lecture relationnelle des stratégies implicites et explicites ainsi que quelques a priori au recours au " public interne » étudiant

\title{
Alain Lavigne
}

\section{OpenEdition}

\section{Journals}

Édition électronique

URL : http://journals.openedition.org/communicationorganisation/2906

DOI : 10.4000/communicationorganisation.2906

ISSN : 1775-3546

Éditeur

Presses universitaires de Bordeaux

Édition imprimée

Date de publication : 1 mai 2004

ISSN : 1168-5549

Référence électronique

Alain Lavigne, "La communication de recrutement a l'université Laval. Une lecture relationnelle des stratégies implicites et explicites ainsi que quelques a priori au recours au « public interne » étudiant », Communication et organisation [En ligne], 24 | 2004, mis en ligne le 27 mars 2012, consulté le 02 mai 2019. URL : http://journals.openedition.org/communicationorganisation/2906 ; DOI : 10.4000/ communicationorganisation.2906

Ce document a été généré automatiquement le 2 mai 2019.

(C) Presses universitaires de Bordeaux 


\title{
La communication de recrutement a l'université Laval. Une lecture relationnelle des stratégies implicites et explicites ainsi que quelques a priori au recours au « public interne » étudiant
}

\author{
Alain Lavigne
}

\section{Introduction}

1 Dans la foulée de plusieurs autres universités nord-américaines qui consacrent d'importantes ressources à la communication de recrutement l'Université Laval se dotait en 1997 d'un premier projet institutionnel de recrutement étudiant. Orchestré autour d'un nouveau vice-rectorat au Développement, le projet s'est concrétisé par l'insertion du recrutement au nombre des priorités institutionnelles, la création d'un Bureau d'information et de promotion (BIP), d'une Table de concertation où siègent des représentants des deux principales associations étudiantes, ainsi que d'une planification stratégique annuelle impliquant une évaluation régulière des orientations et des activités.

2 De fait, dans un contexte hautement concurrentiel entre les universités, d'une démographie défavorable, en plus d'une diminution sensible de ses effectifs étudiants entre 1994 et 1996 l'institution prenait en quelque sorte conscience qu'elle ne pouvait plus compter sur son seul pouvoir d'attraction traditionnel, qui lui avait permis jusque-là de compter sur un nombre toujours croissant d'étudiants pour assurer son développement (VRD. $2000: \mathrm{I})$.

3 Par l'entremise de l'examen du cas de l'Université Laval, la présente communication comportera deux parties. 

considérant que toute communication institutionnelle se déploie de manière non exclusive via six systèmes relationnels. Cette typolologie conçoit que les relations de liens internes et externes misent sur la communication informelle d'affinités (parenté, amitié et partenariat) ouverte à l'interinfluence que les relations de transactions internes et externes misent sur la communication de proximité ouverte à l'interinfluence, alors que les relations de contrôles internes et externes, misent sur la communication marketing peu ouverte à l'interinfluence (Lavigne. 2002).

7 Pour identifier les stratégies implicites et explicites de la communication de recrutement à l'Université Laval, notre analyse visera non seulement la période récente de 1997-2002 alors que les stratégies sont davantage explicites, mais également les années antérieures qui témoignent également de certaines stratégies implicites. D'une part, les quatre premiers plans de recrutement de l'Université Laval nous permettront de dresser un portrait du déploiement des principales stratégies de communication institutionnelle et de ses dernières tendances, notamment un recul des investissements en communication marketing. D'autre part, nous pourrons identifier quatre stratégies implicites qui ont cours depuis la fondation de l'institution et ce. à l'aide de monographies historiques et de l'analyse documentaire.

8 Finalement, afin de dégager quelques a priori au recours au «publie interne » étudiant à des lins de recrutement, notre attention se portera sur l'examen de la campagne de la contre-publicité d'inscription mise de l'avant par les associations étudiantes à l'automne 2002 en réaction à une augmentation importante des frais afférents. Nous avancerons ainsi que le recours aux relations de liens avec le «publie interne » étudiant ne devrait jamais être sollicité de manière aussi instrumentale dans un projet institutionnel.

\section{Les périodes de développement de l'Université Laval}

Fondée en 1852 par l'obtention d'une charte royale accordée au Séminaire de Québec l'Université Laval est la plus ancienne université francophone d'Amérique. La vocation catholique de l'établissement d'enseignement sera confirmée par une bulle papale en 1876. L'historien Jean Hamelin (1995) découpe son évolution en deux grandes périodes, soit de 1852 à 1951, ce qui correspond au son premier siècle où elle se développe lentement dans ses locaux du Vieux-Québec, à proximité du Séminaire, et 1952 à aujourd'hui, où l'établissement connaît une expansion accélérée sur le campus de lu Cité universitaire.

10 Au début de la première période l'Université Laval prendra son essor autour de ses quatre facultés fondatrices: la théologie, le droit, la médecine et les arts. Elle regroupera quelques centaines d'étudiants issus des collèges classiques et se destinant au sacerdoce ou aux professions libérales. Au tournant du XXe siècle, soit en 1901-1902 elle compte 318 étudiants, dont 103 en théologie. 84 en droit. 97 en médecine. 7 en pharmacie et 27 en arts. Selon l'Annuaire général de 1050-1951 quelque 50 ans plus tard l'Université comprend

Communication et organisation, 24 | 2004 
10 facultés. 12 écoles et instituts et une station expérimentale de pêcheries. Elle regroupe alors 118 professeurs et I 720 étudiants.

11 De plus en plus à l'étroit dans le Vieux-Québec, la construction d'un campus s'avérait également justifiée par les prévisions démographiques qui prédisaient, en raison du taux élevé de natalité, que l'établissement aurait à accueillir 3000 étudiants en 1959 et au moins 10000 en 1970. Or la réalité atteindra 3500 en 1956 et 6000 en 1962.

Parallèlement, en 1971 l'établissement adopte de nouveaux statuts qui la détachent de lu tutelle administrative et financière du Séminaire de Québec. L'année suivante, un premier recteur laïc. Larkin Kerwin prend la gouverne de l'Université désormais autonome de l'emprise du clergé.

Les années de croissance allaient se poursuivre jusqu'au milieu des années 90. Un sommet de 36614 inscriptions était atteint en 1992 pour ensuite faire place à une décroissance aux environs de 34000 étudiants entre 1994 et 1996. À l'automne 1998 le nombre d'étudiants franchissait de nouveau le cap des 36000 étudiants, pour ensuite se stabiliser à quelque 35500 étudiants lors des rentrées 1999 et 2000. En 2001 et 2002 les inscriptions atteindront respectivement 35960 et 36665 étudiants, soit un nouveau plafond historique.

Pour les fins de notre étude, nous retiendrons une troisième période de développement, à savoir celle de 1997-2002. Cette dernière est marquée par la structuration formelle du projet de recrutement des clientèles étudiantes.

\section{La situation du recrutement entre 1852 et 1951 : constats communicationnels}

Rien que peu planifiée et avec de faibles ressources, la question du recrutement en est non moins présente au cours de cette période. C'est le Secrétariat de l'université qui est responsable du registre d'inscription et par le lait même, avant la lettre, responsable du recrutement, en publiant entre autres des annuaires d'information. De plus, le clergé catholique agit comme véritable clé de voûte du recrutement, suivant notamment le modèle original des collèges affiliés, dont le Séminaire de Québec au premier chef.

Si le sens commun nous porterait à croire que le recrutement étudiant est peu développé au cours de cette première grande période de l'Université Laval, notre grille d'analyse permet de dégager quelques constats plus nuancés.

\section{Lu stratégie de l'influence informelle omniprésente}

17 De fait, il ressort que la principale stratégie de communication, l'influence indirecte, est la plus appropriée pour rendre compte du recrutement étudiant entre 1852 et 1951. Cette stratégie implicite s'appuie sur les relations de liens entre l'établissement et ses différents partenaires. Elle apparaît comme omniprésente.

Le premier partenaire de ce recrutement est sans contredit le clergé catholique du Québec, qui compte sur un important réseau de liens, en plus de nombreux collèges afin de répondre à ses missions. En quelque sorte, c'est d'abord le Séminaire de Québec qui fournira la « matière première » à « son » Université. Par extension, à compter de 1877 le 
clergé fera profiter l'établissement universitaire de son réseau de collèges du Québec, et même de ceux de l'extérieur, comme autre source efficace de recrutement.

D'autres partenaires sont également sollicités pour le recrutement étudiant au cours de la période, soit les corporations et associations professionnelles, notamment en médecine et droit, ainsi que l'Association générale des étudiants de Laval (ACiLL). à compter de 1929. Ajoutons à cela, ce qu'il ne-tant pas sous-estimer non plus, les relations par les liens familiaux et d'amitié impliquant tous les membres de l'institution d'enseignement, incluant ses dirigeants, ses professeurs, ses étudiants et ses diplômés.

\section{Les stratégies de transactions en soutien}

Tout au long de cette période, la communication de proximité est présente par des stratégies de transactions plus ou moins explicites d'information documentaire, de communication directe et de communication de mécénat.

Ces pratiques communicationnelles propres aux relations de transactions sont assurées par le Secrétariat de l'université. Ses principaux moyens : la production d'annuaires, une communication de première ligne transmise aux étudiants potentiels en quête d'information et l'administration de cinq demi-bourses à l'intention des étudiants désirant étudier la médecine ou le droit.

Ainsi l'Université pratique déjà ce que l'on désigne de nos jours sous le nom de communication de mécénat, soit une stratégie d'action de motivation à une œuvre ou à une personne, apportée sans contrepartie directe de la part du bénéficiaire (Westphalen. 1997: 72). La contrepartie indirecte demeure cependant majeure pour le candidat potentiel, soit une inscription et le complément des coûts de sa première inscription.

Bien qu'il s'agisse des trois stratégies de communication les plus explicites de la période, elles apparaissent toutefois beaucoup plus passives et secondaires par rapport à la stratégie dominante : l'influence informelle par l'entremise des relations de liens.

\section{La situation du recrutement entre 1952 et 1996 : constats communicationnels}

Cette deuxième grande période se caractérise par la croissance accélérée des étudiants et l'intervention de l'Etat dans les affaires universitaires ${ }^{2}$ Un article publié en 1980 dans la revue Affaires universitaires révélait que plusieurs administrateurs d'universités canadiennes n'admettaient alors même pas la nécessité du recrutement (Châteauneuf. 1997). Cette perception pour l'ensemble des universités canadiennes peut s'appliquer au cas de l'Université Laval.

En effet, la promotion du recrutement ne dispose d'aucune ressource humaine et financière spécifique. Tout au plus, le Bureau du registraire assume-t-il son mandat dans la diffusion de l'information générale sur les programmes et les conditions d'admission, en concertation avec le Service des admissions.

Néanmoins, il va sans dire que l'Université s'occupe toujours de façon minimale de son recrutement par certaines pratiques de communication. En 1995, le Rapport du Comité du recrutement au premier cycle brosse un tableau des moyens propres à cette période. 
27 Outre l'information documentaire de masse, le Bureau du registraire organise certaines activités d'information: tournée annuelle des collèges, midis-information, journée d'information à l'Université, visites de l'Université pour les élèves de la région, etc. Le registraire entretient aussi des contacts avec les conseillers en orientation des différents collèges du Québec, par de l'information spécifique et des rencontres particulières.

En 1993 le Conseil d'administration adopte un premier Plan de communication qui précise les clientèles aux lins de recrutement, tout en identifiant certains objectifs généraux. La même année l'Université Laval se dote aussi d'une Politique en matière d'achats de publicité, de commandite et de salons. En 1994 le Service des communications coordonnera, par l'affection d'une nouvelle ressource professionnelle spécialisée (Rapport annuel. 1994) sa première grande action publicitaire de recrutement à l'échelle du Québec. Cette campagne visera exclusivement à susciter les inscriptions aux études de $2 \mathrm{e}$ et $3 \mathrm{e}$ cycles. Un conseiller en relations de presse sera aussi embauché (Rapport annuel. 1995). Le système d'information électronique Alérion sera inauguré en novembre 1994.

Dans la foulée de ces premiers pas en matière de recrutement l'Université amorcera une réflexion en profondeur sur cette problématique. Du côté des partenariats institutionnels, rappelons-le cette période marque la lin de la tutelle avec le Séminaire de Québec, ce qui relègue les liens entre l'Université et le clergé à un niveau plus distancié. Cependant, deux nouveaux partenaires d'importance se positionneront comme des acteurs susceptibles de soutenir le recrutement, soit l'Association des diplômés de l'Université Laval (ADUL) créée en 1948 et l'Union des gradués de Laval (l'UGIL) créée en 1967.

30 Cette seconde période, surtout lors de ses dernières années, se caractérise par une amorce de réflexion et le déploiement des premières ressources humaines et financières destinées au recrutement. Pour l'année 1996-1997 si on additionne les montants investis au recrutement en faculté et ceux qui le sont au niveau institutionnel, incluant les salaires, cela représente toutefois moins île l'«du budget de fonctionnement de l'Université (VRD. 1998 : 12).

\section{Les stratégies de transactions dominantes}

31 Au chapitre de la communication, contrairement à la première période, les relations dominantes nous apparaissent celles de transactions, par le biais des stratégies d'information documentaire, de communication directe et de communication de mécénat, en plus des nouvelles stratégies ${ }^{3}$ de communication événementielle, de relations de presse, de communication électronique et d'influence formelle.

L'information documentaire ${ }^{4}$ et les contacts avec les étudiants potentiels sont désormais organisés pour répondre à des besoins de masse. De plus l'Université maintient sa stratégie de communication de mécénat, en structurant son offre de bourses d'admission ${ }^{5}$ , tout comme le fera également l'ADUI, sur une base annuelle ${ }^{6}$

33 Pour ce qui est de la stratégie de communication événementielle, elle prend la forme d'une tournée annuelle des collèges, des midis-information, la journée d'information à l'Université et les visites de l'Université pour les élèves de la région. L'embauche d'une ressource spécialisée en relations presse ${ }^{7}$ et la mise en ligne du site Alérion ouvrent de nouvelles avenues stratégiques en matière d'information médiatique et de communication électronique. 

annuel de recrutement et de faire rapport sur les orientations pour l'année qui suit le plan. Deux personnes sont affectées à la coordination des activités de recrutement, l'une à l'externe et l'autre à l'interne, de même que l'organisation en 1998 d'un Bureau d'information et de promotion (BIP). Pour l'année 2000-2001 l'équipe du personnel régulier du BIP comptait une directrice, six professionnels et quatre employés de soutien. Le budget du BIP est destiné principalement à la réalisation des différentes activités de promotion, frais de poste et production de diverses publications. À l'automne 1999 le BIP se dote d'un bureau à Montréal.

40 demandes d'admission aux premier, deuxième et troisième cycles. Du même coup, le territoire de recrutement s'étend non seulement à l'ensemble du Québec mais aussi hors 
Québec. A cette fin sont notamment augmentées l'offre de bourses ainsi que la participation à des salons $\mathrm{cl}$ à des tournées d'établissements d'enseignement situés tant au Québec qu'à l'extérieur. Quelques nouveaux partenariats sont développés.

41 Contrairement aux deux autres périodes analysées, cette troisième période se démarque par une planification stratégique formelle, la spécialisation de ressources humaines et des budgets annuels spécifiques destinés au recrutement. Les relations de contrôles deviennent celles qui monopolisent le plus de ressources financières, avec leurs stratégies de publicité, de promotion et d'image de marque. Les relations de transactions progressent aussi, entre autres pur une nouvelle stratégie de communication interne. Les relations de liens demeurent toujours présentes en filigrane.

\section{Des stratégies de communication marketing affirmées}

42 L'enveloppe budgétaire affectée à la stratégie publicitaire sera en progression continue au cours de la période, passant de 314500 \$. en 1998-1999. à 367000 . en 2001-2002. On justifie le recours à cette stratégie dans le plan de 1998 (p.7) en précisant que l'investissement publicitaire en soutien au recrutement doit augmenter afin de répondre aux vœux de la Commission d'orientation et soutenir efficacement cette priorité institutionnelle. Dans cette lignée communication marketing, un nouveau poste budgétaire sera consacré aux « objets promotionnels » à compter du plan 1999-2000 soit l'ajout d'une nouvelle stratégie de promotion.

De son côté, la préoccupation pour une stratégie d'image de marque ${ }^{9}$ apparaît en 1998 via une orientation touchant la visibilité de l'Université Laval, par la diffusion d'une image cohérente, la mise en valeur des caractéristiques distinctives des programmes et des avantages concurrentiels (VRD. $1998: 5$ ).

\section{Des stratégies de transactions bonifiées}

En plus des stratégies d'information documentaire, de communication directe et de communication de mécénat présentes depuis la première période 1852-1951 qui seront encore bonifiées tant au plan documentaire ${ }^{10} q u$ 'électronique ${ }^{11}$, la dernière période associe formellement les publies internes aux efforts de recrutement.

Une orientation du premier plan de recrutement de 1998 identifie la communication interne en vue d'assurer la mobilisation, la coordination nécessaire et l'ajustement des messages véhiculés (VRD. 1998 ; 5). Dés l'automne 1997, la Table de concertation sur le recrutement étudiant, agira comme lieu privilégié d'échange d'information, de mise en oeuvre des orientations institutionnelles et facultaires ainsi que la coordination de l'ensemble des activités de recrutement. À un autre niveau, le Groupe des associés au développement de l'Université Laval (GADUL), mis sur pied en 1999, servira cette même fin.

De son côté, la stratégie de communication événementielle sera raffinée par la création de nombreux événements internes, notamment des opérations portes ouvertes et la participation systématique à des événements externes. Pour le plan 2000-2001. à titre d'exemple, l'annexe consacrée à ce sujet compte quatre pages.

47 Quant à la stratégie d'influence directe, elle ne se limitera plus aux conseillers des collèges. Elle s'étendra à l'ensemble des conseillers provenant des autres régions du 
Canada. «Pour ce qui est de l'international, les principaux contacts sont établis par l'intermédiaire des Centres d'éducation canadiens, les ambassades canadiennes situées dans les pays-cibles ainsi que certains organismes voués à l'éducation (CREPUQ. NACAC.NASA. etc.) constituent aussi d'excellents intermédiaires pour nous aider à atteindre les groupes visés. » (VRD. 1999 : 13-14)

Enfin, comme nous l'avons souligné, la communication de mécénat est augmentée au cours de la période. L'usage stratégique des bourses est confirmé dans le plan 2000-2001. alors qu'on vante les bienfaits de «l'utilisation des bourses disponibles à des lins stratégiques de recrutement de manière à garantir un soutien financier à un nouvel étudiant »(p.11).

\section{Lu stratégie d'influence informelle toujours en filigrane}

49 Tous les grands partenaires traditionnels identifiés pour les périodes précédentes demeurent actifs pour la présente période, En filigrane, ils continuent à jouer leur rôle dans ce que nous avons nommé la stratégie d'influence informelle. Entre autres nouveaux partenaires, relevons le Club des ambassadeurs. Mise sur pied en 1997 cette entité a pour mission d'impliquer directement des bénévoles et des étudiants dans le recrutement de l'établissement. Par ailleurs, il est permis d'avancer à l'élargissement du bassin de partenaires relais, notamment pour le recrutement à l'étranger. Les clubs d'anciens de l'ADUL. à l'étranger constituent des exemples patents d'entités de relations de liens utiles au recrutement. « Au cours des deux dernières années, nous avons mené plusieurs actions conjointes, tant au Québec qu'à l'extérieur... Il sera essentiel, au cours des années à venir, de maintenir et même d'intensifier ce travail de collaboration dont les effets sur le recrutement ne peuvent être que positifs. » (VRD. 2002 : 14).

\section{Ressources financières et stratégies de communication : quelques tendances}

50 Les postes budgétaires identifiés dans les quatre plans de recrutement nous permettent de dégager certaines tendances en matière de publicité, de promotion, d'information documentaire, de communication événementielle et d'influence formelle.

51 Comme nous l'avons déjà relevé, la croissance continue des sommes affectées à la stratégie publicitaire est manifeste. Cependant, en proportion de l'ensemble des dépenses consenties au recrutement, l'évolution de ce poste budgétaire accuse un recul constant avec des pourcentages respectifs de $43.4 \%$. en 1998-1999 de 40.9\%. en 1999-2000 de $35 \%$. en 2000-2001. et de $32 \%$. en 2001-2002. Il en est ainsi pour les nouvelles sommes injectées en promotion. Ces reculs sont justifiés par une réserve vis-à-vis un usage abusif des pratiques du marketing exprimée dans le plan 1999-2000. à savoir que le marketing «ne viendra pas remplacer l'intérêt d'informer sur nos programmes et sur nos activités, de les promouvoir, de les publiciser (sie), mais il entraîne une façon différente de le faire » (p. 21).

52 Par ailleurs, l'information documentaire accuse aussi un important recul. Le recours à la communication électronique à plus large échelle en est certainement pour quelque chose. Dans son plan 2000-2001 le vice-recteur au Développement rapporte les résultats d'une étude américaine qui révèle que $90 \%$ des étudiants d'établissements pré-universitaires 
qui sont en quête d'un programme d'étude ou d'une université ont recours aux sites Web (p. 12).

Pour sa part, la communication événementielle, qui monopolisait $18.4 \%$ du budget en 1998-1999. a vu ce pourcentage hausser à 19.5 en 1999-2000 à 20.6. en 2000-2001 et à 24.8. en 2001-2002. Cette stratégie est la seule qui gagne constamment du terrain depuis le premier plan de recrutement de 1998-1999.

Enfin, il est à noter que l'influence informelle dispose d'un petit budget stable autour de $0.5 \%$. Sont comptabilisées dans ces dépenses, les activités « pour bien l'aire passer notre message, de tenir compte des partenaires (conseillers en orientation, conseillers en information scolaire et professionnelle, professeurs, etc.). Ils constituent notre prolongement et contribuent à la permanence de notre présence au sein de différents groupes » (VRD. 1998 : 10).

\section{Quelques a priori au recours au «public interne » étudiant}

55 En matière de communication de recrutement étudiant, la période récente 1997-2002 dénote clairement une volonté de coordination plus systématique avec l'ensemble des publies internes. Le lieu principal de mise en œuvre des orientations institutionnelles et facultaires en devient dès lors la Table de concertation, instance conseil regroupant une quarantaine de personnes. Outre des représentants du vice-rectorat au Développement et de toutes les unités de l'institution, on y retrouve un représentant de la Confédération des associations d'étudiants et d'étudiantes (CADEUL) et un autre de l'Association des étudiâmes et des étudiants de Laval inscrits aux études supérieures (ALLIES). Ces derniers constituent, en quelque sorte, des représentants du «publie interne » étudiant, dont la collaboration opérationnelle et fonctionnelle est sollicitée en raison de leurs relations de liens avec le projet institutionnel.

Dans un contexte non conflictuel, cette collaboration étudiante ne pose aucun problème. Toutefois dans le contexte de la rentrée de l'automne 2002 la situation prendra une dimension inattendue. En effet, les deux associations étudiantes seront alors les promoteurs d'une campagne de contre-publicité d'inscription, en réaction à de nouveaux frais afférents de 180 \$ imposés par l'institution à tous les étudiants inscrits à temps plein. Or dès la convocation de la Table de concertation d'octobre, le vice-recteur au Développement procédera à l'exclusion des représentants des étudiants. Dans son courriel aux deux personnes concernées, il écrit qu'il lui semble préférable de ne plus les inviter aux travaux de l'instance « par respect des convictions des uns et des autres. La campagne de publicité négative menée par la CADEUL et L'AELIES est incompatible avec les objectifs de la Table de concertation dont j'ai la charge » (LaFerrière. 2002).

Comment expliquer une décision aussi radicale de la part du représentant de l'institution?

Selon notre grille d'analyse relationnelle, le vice-recteur au Développement était en droit de s'attendre de la part des représentants du «public interne " étudiant à une collaboration de liens envers le projet institutionnel. Toutefois, ces derniers ont également le mandat de veiller à la défense des intérêts des étudiants dans tous ses rapports sociopolitiques avec l'institution. Un mandat qui les place d'abord et avant tout dans un système relationnel de transactions. 

certaines manifestations de cette communication bien avant la structuration de cette mission en 1997. A l'aide de la grille relationnelle qui nous a guidée, nous avons pu non seulement identifier les stratégies explicites de communication pour la période récente de 1997 à 2002 mais également certaines stratégies implicites présentes depuis la fondation de l'institution, en 1852.

De plus, il nous a aussi été possible de dégager quelques a priori au recours au «publie interne » étudiant dans une telle démarche de recrutement. À cet effet, l'examen de la campagne de la contre-publicité d'inscription mise de l'avant par les associations étudiantes à l'automne 2002 en réaction à une augmentation importante des frais afférents, nous a permis d'avancer que le recours aux relations de liens avec le «publie interne » étudiant ne devrait jamais être sollicité de manière aussi instrumentale dans un projet institutionnel. 
De fait, si la coexistence de divers acteurs dans un projet institutionnel gagne généralement à miser à la fois sur des relations de liens et de transactions, dans un contexte conflictuel, cependant, les unes peuvent parfois empêcher les autres!

\section{Ouvrages et articles}

\section{Rapports et documents}

Annuaire général 1935-1954. Québec : Université Laval. 1933.

Annuaire général 1950-1951. Québec : Université Laval. 1950.

Annuaire général 1952-1953. Québec : Université Laval. 1952.

Annuaire général 1975-1976. Québec : Université Laval. 1975.

Plan de communication de l'Université Laval. Conseil d'administration, février 1993.

Rapport annuel 1993-1994. Québec, Université Laval. 1994.

Rapport annuel 1994-1995. Québec. Université Laval. 1995.

Rapport de la Commission d'orientation de l'Université Laval. Conseil

d'administration, mars 1998.

Rapport du Comité d'orientation du recrutement au 1er cycle, Université Laval, janvier 1995.

Rapport pour l'année académique 1970-1971. Université Laval. Bureau du secrétaire général. 1971.

Renseignements généraux 1995-1997. Université Laval. Mai 1995.

Service des communications. Plan de relations de presse 1995-1996.

Université Laval, juin 1995. 4 pages et annexes.

Service des communications. Plan de relations de presse 1996-1997.

Université Laval, juin 1996. 4 pages et annexes.

Service des relations extérieures. Mandat. Conseil universitaire, décembre 1949.

Vice-rectorat au Développement (VRD). Plan de recrutement des effectifs étudiants à l'Université Laval. 1997-1998 et 1998-1999. Conseil d'administration de l'Université Laval, avril 1998.

Vice-rectorat au Développement (VRD). Rapport de l'implantation du Plan de recrutement des effectifs étudiants à l'Université Laval. 1997-I998 et 1998-1999 et orientations pour l'année 1999-200 (1. Conseil d'administration de l'Université Laval, avril 1999.

Vice-rectorat au Développement (VRD) Le recrutement étudiant en 1999-2000 et les orientations 2000-2001. La première étape du processus de suivi des étudiantes el des étudiants. Conseil d'administration de l'Université Laval, avril 2000.

Vice-rectorat au Développement (VRD) Le recrutement étudiant : bilan 2000-2001 et orientations 2001-2002. La première étape du processus de suivi des étudiantes et des étudiants. Conseil d'administration de l'Université Laval, avril 2001.

Vice-rectoral au Développement (VRD). Le recrutement étudiant: bilan 1997-2002 et orientations La première étape du processus de suivi des étudiantes et des étudiants. Conseil d'administration de l'Université Laval, mars 2002. 


\section{BIBLIOGRAPHIE}

CHATEAUNEUF, I... Quand les étudiants sont des clients. Nouvelles approches en recrutement universitaire. Affaires universitaires, août-septembre 1997.

CUTLIP ». S.M.. Advertising Higher Education. In CUTLIP. S.M. History of Public Relations, from the $17^{\text {th }}$ to the $20^{\text {th }}$ Century: tlie Antecedents. Hillsdale : Lawrence Erlbaum. 1995. p. 229-251.

GAUDREAU. V.. Au \grands maux les grands remèdes. L'ALLIES porte la cause devant les tribunaux. l'Exemplaire. 14 janvier 2003. HAMLLIN..J.. Histoire de l'Université Laval. Sainte-Foy : Les Presses de l'Université Laval. 1995.

LAFERRIERE. M.. Etudiants chassés de la concertation. L'Université Laval invoque leurs divergences sur les frais afférents. Le Soleil. $1^{\text {er }}$ octobre 2002. p. 9.

LAVIGNE A.. La communication institutionnelle vue par l'entremise de six grands systèmes relationnels : proposition d'une typologie. Communication \& Organisation. I er semestre 2002. p. 155-173.

LEMIEUX. A. (sous la direction de) L'organisation de l'éducation au Québec. Structure et fonctionnement. Montréal : Editions Agence d'Arc. 1992.

NGUYEN. N.. Éléments d'information contribuant à la formation de l'image d'un établissement universitaire. Revue canadienne de l'éducation. 19. 4. 1994. p. 386-398.

WESTPHALEN. M.-H. La communication externe de l'entreprise. Paris : Dunod. 1997.

\section{NOTES}

1. Cette partie de la présente contribution a fait l'objet d'une communication lors du congrès 2 (103 de l'Association francophone pour le savoir (ACFAS), tenu à Rimouski (Canada) Des résultats plus détaillés seront également publies dans la revue Communication

2. Au Québec, a compter de 1964 l'État est intervenu par la création du Conseil des universités (1968) par la création de l'Université du Québec (1968). par le financement des universités et par la Direction générale du ministère de l'Education. "Si les chartes de corporations privées subsistent, les universités sont de plus en plus traitées comme des services publics. Par un financement décisif par la révision des chartes, par le conseil des universités l'Etat coordonne et oriente désormais l'enseignement universitaire » (Lemieux. 1992 :373).

3. La création d'un Service des relations extérieures, a compter de 1949, contribuera au déploiement plus systématique de la communication événementielle et des relations de presse, en vue d'« étendre le renom de l'Université I aval et faire connaître dans tous les milieux les services qu'elle rend (Service des relations extérieures. 1049) En 1965, a la faveur d'un nouveau mandat, cette unité deviendra le Service des relations publiques, qui prendra la présente appellation de Service des communications en 1992

4. La création d'un Service des relations extérieures, a compter de 1949, contribuera au déploiement plus systématique de la communication événementielle et des relations de presse, en vue d'« étendre le renom de l'Université I aval et faire connaître dans tous les milieux les services qu'elle rend» (Service des relations extérieures. 1049) En 1965, a la faveur d'un nouveau 
mandat, cette unité deviendra le Service des relations publiques, qui prendra la présente appellation de Service des communications en 1992

5. La création d'un Service des relations extérieures, a compter de 1949, contribuera au déploiement plus systématique de la communication événementielle et des relations de presse, en vue d'« étendre le renom de l'Université I aval et faire connaître dans tous les milieux les services qu'elle rend» (Service des relations extérieures. 1049) En 1965, a la faveur d'un nouveau mandat, cette unité deviendra le Service des relations publiques, qui prendra la présente appellation de Service des communications en 1992

6. La création d'un Service des relations extérieures, a compter de 1949, contribuera au déploiement plus systématique de la communication événementielle et des relations de presse, en vue d'« étendre le renom de l'Université Laval et faire connaître dans tous les milieux les services qu'elle rend " (Service des relations extérieures, 1949 En 1965. a la faveur d'un nouveau mandat cette unité deviendra le Service des relations publiques, qui prendra la présente appellation de Service des communications en 1992

7. La création d'un Service des relations extérieures, a compté de 1949, contribuera au déploiement plus systématique de la communication événementielle et des relations de presse, en vue d'« étendre le renom de l'Université Laval et faire connaître dans tous les milieux les services qu'elle rend» (Service des relations extérieures. 1949). En 1965. a la laveur d'un nouveau mandat, cette unité deviendra le Service des relations publiques, qui prendra la présente appellation de Service des communications en 1992

8. La création d'un Service des relations extérieures, a compté de 1949, contribuera au déploiement plus systématique de la communication événementielle et des relations de presse, en vue d'« étendre le renom de l'Université Laval et faire connaître dans tous les milieux les services qu'elle rend ( (Service des relations extérieures. 1949). En 1965. a la laveur d'un nouveau mandat, cette unité deviendra le Service des relations publiques, qui prendra la présente appellation de Service des communications en 1992

9. La création d'un Service des relations extérieures, à compter de 1949, contribuera au déploiement plus systématique de la communication événementielle et des relations de presse, en vue d'« étendre le renom de l'Université Laval cl faire connaître dans tous les milieux les services qu'elle rend» (Service des relations extérieures. 1949) In 1965. a la faveur d'un nouveau mandat, cette unité deviendra le Service des relations publiques, qui prendra la présente appellation de Service des communications en 1992.

10. La création d'un Service des relations extérieures, à compter de 1949, contribuera au déploiement plus systématique de la communication événementielle et des relations de presse, en vue d'« étendre le renom de l'Université Laval cl faire connaître dans tous les milieux les services qu'elle rend (Service des relations extérieures. 1949) In 1965. a la faveur d'un nouveau mandat, cette unité deviendra le Service des relations publiques, qui prendra la présente appellation de Service des communications en 1992.

11. Les premières actions de publicité de masse associées a l'Université lavai remontent a 1920 et a 1448. a l'occasion des deux premières campagnes de souscriptions publiques de l'institution (Hamelin. 1995 : 208-209, Côté programmes les premières actions se feront a compter de 1962 pour les cours para-universitaires offerts aux adultes (Hamelin. 1995 227) Aux États-Unis, plusieurs collèges et universités recourant a la publicité de masse des le début $\mathrm{du} \mathrm{xx}^{\mathrm{e}}$ siècle, non seulement pour des tins de souscriptions publiques mais aussi de promotion des programmes (Cutlip, 1995 2.1I-232) 


\section{AUTEUR}

\section{ALAIN LAVIGNE}

Université Laval. Québec, Canada 